\title{
Ease of use and validity testing of a point-of-care fast test for parasitic vaginosis self-diagnosis
}

\author{
Hawash, Y. ${ }^{1 *}$, Jaafer, N. ${ }^{2,3}$, Alpakistany, T. ${ }^{4}$ \\ ${ }^{1}$ Department of Microbiology, College of Medicine, Taif University, P.O. Box 11099, Taif 21944, Saudi Arabia \\ 2Department of Molecular and Clinical Parasitology, National Liver Institute, Menoufia University, Menoufia, Egypt \\ ${ }^{3}$ Department of Obstetrics and Gynecology, King Faisal Medical Complex, Taif, KSA \\ ${ }^{4}$ Department of Microbiology, King Faisal Medical Complex, Taif, KSA \\ *Corresponding author: y.hawash@tu.edu.sa
}

\section{ARTICLE HISTORY}

Received: 1 August 2021

Revised: 19 October 2021

Accepted: 19 October 2021

Published: 21 December 2021

\begin{abstract}
There is a demand for patients to self-diagnose their sexually transmitted infections (selftesting), particularly during the coronavirus pandemic to prevent infection spread. We enrolled a cohort of Saudi women in a single-visit prospective study, which was the first of its kind performed in the country. Our aim was to evaluate the OSOM $^{\circledR}$ Trichomonas (OSOM) $^{\circ}$ test, a single-use, point-of-care rapid test, for its efficacy and accessibility as a self-test for Trichomonas vaginalis (Trichomonas) infection. At a public hospital's gynecology clinic, women received sufficient training on specimen collection and OSOM self-testing. The women's infection status was re-evaluated using direct wet mount microscopy and clinician performed OSOM using additional swabs. Specimens with discordant results were sorted using an inhouse polymerase chain reaction (PCR). 174 women aged 18 to 35 were registered and selftested at the clinic under the supervision of a gynecologist between June and December 2020 , with 84.4 percent (147/174) having a valid result on the first or repeat OSOM. Infection was detected in $12.2 \%(18 / 147)$ of participants, with two-thirds of them symptomatic. Young age, low education, the existence of vaginitis symptoms, and unemployment were identified as key risk factors for infection in the study population, with statistically significant differences seen among women only in terms of education level and employment status $(p<0.001)$. The OSOM self-test performed well $(83.3 \%$ sensitivity and $98.4 \%$ specificity), outperforming the wet mount microscopy $(72.2 \%$ sensitivity and $100 \%$ specificity) and comparable to the clinicians' OSOM ( $88.8 \%$ sensitivity and $100 \%$ specificity). The patients' and clinicians' OSOM tests were strongly correlated, with a kappa of 0.89 and a $97.9 \%$ agreement. Self-collection of vaginal swabs was accepted and preferred by most women (94\%) over the clinician-collection. Overall, our study's findings may have important consequences for the implementation of Trichomonas screening based on OSOM self-testing approach in the study's population.
\end{abstract}

Keywords: OSOM Trichomonas self-test; diagnostic performance; accessibility; coronavirus pandemic.

\section{INTRODUCTION}

Trichomoniasis is an infection caused by the parasite Trichomonas vaginalis (Trichomonas). Trichomonas is a singlecelled motile protozoa with no cyst form. Only humans are known to be infected with Trichomonas (Lewis, 2014). Infection is transmitted primarily through sexual contact, but nonsexual transmission was also reported (Crucitti et al., 2011). Infection usually affects women's lower genital tracts and men's urethras. Infection affects 153 million individuals worldwide, which is more than the combined number of chlamydia and gonorrhea, the two most common sexually transmitted infections (STI) as described by Manhart (2017).
Trichomonas infections usually occur with no symptoms, which means that regular screening for infection is important. The symptoms can range from slight discomfort to severe inflammation. Itching, burning while urinating, and abnormal discharge are all possible symptoms for Trichomonas vaginosis. An infected partner, whether symptomatic or not, can nonetheless spread the infection to others (Maciel et al., 2004). If not treated properly, infection can cause pelvic inflammatory disease, early rupture of membranes, premature labor, low birthweight, post-abortion infections, and an increased risk of the human immunodeficiency virus (HIV) as reported by Masha et al. (2019). 
Microscopic examination of vaginal secretion or swabs "wet mount" is often adopted for routine diagnose of Trichomonas infection. Nucleic acid amplification tests (NAAT) and rapid antigen detection (RAD) tests, two more sensitive assays, are also available but not for the routine diagnosis. Culture, as sensitive and specific diagnostic method, could be used for infection confirmation in research. Even in research, culture has been overtaken as the gold standard by NAATs being the most sensitive diagnostic approach (Hobbs \& Seña, 2013).

Trichomoniasis is thought to be underdiagnosed in women for a variety of reasons: To begin with, most of infections occur in populations lack access to diagnosis and treatment services. Second, even when services are available, it is frequently considered inadequate, and of low-quality. Barriers like concerns about autonomy, discomfort, stigma, and loss of privacy deter high risk populations and others from seeking STI testing at a health-care provider or a clinic (Lee \& Cody, 2020). The wet mount, the most commonly used method in routine diagnosis is frequently blamed from being of low sensitivity, in comparison to other methods (Nabweyambo et al., 2017). Lastly, in certain circumstances such as during humanitarian crises like the coronavirus pandemics the routine health services for STIs is disrupted and the existing health systems is overburdened (Napoleon et al., 2020).

At the time of coronavirus pandemic, integrating selfcare "self-testing" into STIs health services can be a useful tool to boost the primary health care service, ensure its continuity and prevent coronavirus infection spread. Selftesting can be offered entirely or partially outside of routine health services and used with or without the direct supervision of health care personnel as it has been mentioned by Gaydos (2018). The world health organization has issued worldwide normative recommendations on self-care activities such as self-testing for STIs (WHO, 2020). Two Food and Drug Administration (FDA)-approved point-ofcare tests, Affirm VP III (BD Diagnostic Systems, Sparks, MD) and OSOM Trichomonas rapid test (Genzyme Diagnostics, Cambridge, MA), are now available, with potentials allowing users to collect and test their own samples (Herbst de Cortina et al., 2016). For the first time in Saudi Arabia, we conducted a prospective study to evaluate the performance and acceptability of the Trichomonas self-diagnostic test among young women. The OSOM ${ }^{\circledR}$ Trichomonas Rapid Test performance was evaluated on both self-collected and clinician-collected vaginal swabs as a clinic-based program, and the results were compared. The results were compared in view of true positives defined as PCR and/or wet mount microscopy positives.

\section{MATERIALS AND METHODS}

\section{The study Protocol}

A prospective study was undertaken in king Faisal General hospital, at Taif district, western Saudi Arabia between June and December 2020. Women during a visit to the gynecology clinic were given an overview of the ongoing research protocol and invited to participate. Women who agreed to participate in the study were questioned for their basic sociodemographic characters, presenting symptoms and their awareness about STIs. Women who deemed to be eligible for the study, were provided with clear information on how to correctly collect a vaginal swab sample on their own and on how to do a self-test using the OSOM kit.

\section{Inclusion/exclusion criteria}

To meet the inclusion criteria, women had to be $18-35$ years, educated and willing to conduct a speculum examination. Illiterate women under the age of 18 , currently menstruating, pregnant, unwilling to do a pelvic examination, and/or who had symptoms requiring emergency gynecological care or had used vaginal douches within two days of interviewing or had recent ( 15 days) metronidazole intake were excluded from the study. After menstruation, menstruating women were allowed to participate in the study. Healthcare professionals were also excluded from studying. Women who fit the above eligibility criteria were approached to inquire about their interest in participating in the research study.

\section{Concerns about ethics}

The research proposal was evaluated and authorized by the institutional ethical committee under the IRB (Ref: KFMC-02T-067). Women had to offer informed consents to take part in the study. Personal information about participants was not recorded in order to maintain anonymity. Women were also told that they might exit the research at any time and would face no consequences. Women with positive results were followed-up by the gynecologist and treated.

\section{Demographic and clinical data collection}

Women characterized themselves as symptomatic or asymptomatic based on the presence or absence of vaginitis-associated symptoms (discharge, irritation, itching, dysuria, and/or dyspareunia). After that, women were asked regarding the basic socio-demographic characters (age, marital status, educational level, residency, employment status and nationality) and about their information regarding STIs (common types, prevention, diagnosis, POC tests and pregnancy test).

\section{Specimen collection and processing}

Swabs for vaginal specimen collection were supplied to eligible women who agreed to participate. Women received enough instruction on how to collect their own specimens and perform the test. In the clinic, and under the supervision of the gynecologist, each woman collected one vaginal swab following the given instructions. Briefly, each participant was told to insert a sterile rayon swab from the kit two inches into the vagina, swirl three times along the upper vaginal wall, then to remove and place the swab into the collecting tube with a buffer.

In addition, each woman was asked to do a pelvic examination and additional three vaginal swabs were collected via a female gynecologist: one swab put into collection tube containing $1 \mathrm{ml}$ extraction buffer for the clinician performed OSOM, a second swab placed into a collection tube containing $0.5 \mathrm{ml} 0.9 \%$ saline for the routine wet prep microscopy and the third swab was inserted into an empty collecting tube and kept frozen at $-20^{\circ} \mathrm{C}$ for PCR assay.

\section{Specimen examination}

\section{OSOM $^{\circledR}$ Trichomonas Rapid Test}

In the clinic on the same day, both women and the clinician did test for the corresponding swabs with the OSOM rapid test following the kit's protocol. Briefly, patient or the clinician, each has to squeeze liquid from swab while removing it from the solution. Then, she threw away the swab, and added the test strip into the buffer solution. After 10 minutes, she read the test results. The test is considered 
negative if just the internal control line (red) was visible and positive when both the red control line and the test line (blue) were visible. If both the red control line was absent, the test was declared as invalid and has to be repeated.

\section{Direct wet prep microscopy}

In the clinician's office, a drop of the saline-swab combination was deposited on a clean glass slide with cover and inspected microscopically for motile Trichomonads under 100x and 400x microscopy within $30 \mathrm{~min}$ of collection as routinely done at the clinic. Presence of motile trichomonads indicated a positive result for Trichomonas infection (Garber, 2005).

\section{Trichomonas DNA extraction}

In the Microbiology laboratory, extraction of Trichomonas genomic DNA (if present) was performed using the traditional phenol chloroform method. Briefly, each swab was dipped in sterile $10 \%$ sodium dodecyl sulphate solution contained in a $1.5 \mathrm{ml}$ tube, and heated for $5 \mathrm{~min}$ at $65^{\circ} \mathrm{C}$. The swabs were then squeezed against the wall of the tube to express fluids. A volume of $100 \mu \mathrm{l}$ of sodium acetate was added to neutralize the alkaline component of the lysis solution. A volume of $600 \mu \mathrm{l}$ of phenol chloroform and $25 \mu \mathrm{l}$ distilled water was then added. The mixture was vortexed and span at maximum speed $(14000 \mathrm{rpm})$ for $30 \mathrm{~min}$. Purification of extracted DNA was achieved by precipitation using absolute isopropanol. Final DNA precipitate was dissolved in $50 \mu \mathrm{l}$ of nuclease-free water and stored at $-20^{\circ} \mathrm{C}$ until amplification.

\section{Trichomonas DNA amplification}

A set of primers designed to target 290 base pair of a highly conserved region in the AP65 adhesin genes of Trichomonas, was used in PCR amplification, as described elsewhere by Alderete et al. (1995). The PCR reactions were set up in a final volume of $25 \mu \mathrm{l}$ with reagents concentrations and the temperature cycling conditions very close to that mentioned in an earlier protocol (Nabweyambo et al., 2017). A gradient thermocycler was used to carry out the amplification reactions. The PCR products were visualized and analyzed on a $1.5 \%$ agarose gel electrophoresis stained with ethidium bromide.

\section{Women's opinions on the OSOM self-test}

A seven-points-based simple survey was undertaken to determine women's views and acceptability of the OSOM self-testing in comparison to the clinician-testing. The survey was used to determine whether women understood the test's instructions, swab collection procedure, the test results, re-use of OSOM if made available for the public, recommendation of the test to others, and whether they prefer home testing over clinic testing or vice versa.

\section{Data collection and analysis}

Computer software SPSS, version 21 (Chicago, IL, USA), was used to collect, tabulate, and analyze the research data. We used descriptive frequencies to define our population and we ran chi-square $(\chi 2)$ test analyses of association between the OSOM self test results and women's characteristics. Based on the results of an in-house PCR, which was designated as the gold standard in our study, the diagnostic performance characteristics of self-testing, clinician-testing and wet mount microscopy, in terms of sensitivity, specificity, positive predictive value (PPV), and negative predictive value (NPV) were calculated. We compared the correlation between the self and clinician-rapid tests using a kappa statistic. We calculated point estimates and $95 \%$ confidence intervals (95\% $\mathrm{Cl})$ for sensitivity and specificity for each diagnostic test method. We categorized the data into variables based on criteria (presence or absence) to see if these variables influenced test performance (sensitivity or specificity).

\section{RESULTS}

Over a 6-months period, 320 women were interviewed, 274 expressed their interest to participate in the study, 174 of whom met the inclusion criteria. Of these, only 147 (84.4\%) women had a valid result on either the first or repeat test. These participants- comprising the study sample- were subjected to pelvic examination and completed data in baseline and post-testing questionnaires (Figure 1).

Table 1 shows the sociodemographic characteristics of the women who took part in the study. The median age of the participants was 23.3 years (IQR: $18-35$ ). The majority of them were married $(73.4 \%, 108 / 147)$, had completed at least 9 years of school $(81.6 \%, 120 / 147)$, worked $(80.9 \%$, $119 / 147)$, and resided in the city $(63.9 \%, 94 / 147)$. Participants' knowledge of STIs and attitudes toward OSOM self-testing were exhibited in Table 2. The vast majority of women (83.6\%) had never heard of STIs before, were unfamiliar with STIs preventive tools $(80.9 \%)$, and wanted to keep STIs test results private $(79.5 \%)$. Three out of every five women $(59.1 \%)$ have never done pregnancy test by their own before, and almost everyone has never heard of the Trichomonas Rapid test.

Based on the in-house PCR assay results, the diagnostic performance characteristics of the different tests were calculated as shown in Table 3 . The overall prevalence of $T$. vaginalis infection was $12.2 \%(18 / 147)$ of the studied population. For the patient-collected vaginal swab specimens, the OSOM exhibited sensitivity and specificity of $83.3 \%$ and $98.4 \%$, respectively. For the clinician-obtained vaginal swab samples, the OSOM exhibited sensitivity and specificity of $88.8 \%$ and $100 \%$, respectively. In contrast, the microscopic examination of wet mount preparations showed sensitivity and specificity of $72.2 \%$ and $100 \%$, respectively. As could be seen in Table 4, both the OSOM on patient- and cliniciancollected vaginal specimens were highly correlated, with 97.9\% agreement and a calculated kappa coefficient value of 0.89 . The overall agreement between the OSOM self-testing and the wet prep microscopy was $97.2 \%$ with reported Cohen's kappa value of 0.85 .

We looked into which characteristics of women were linked to Trichomonas infection as seen in Table 1. Infection was found to be more common among low-educated than among middle- to high -educated women, with a significant difference observed $(p<0.001)$. In addition, infection was discovered more frequently in non-working than in working women, with a statistically significant difference recorded $(p<0.001)$. Aside from these two characteristics, there were no significant associations between Trichomonas infection and the women's age, the presence or absence of vaginal symptoms, the patients' nationality, or their place of residence.

Lastly, the women's responses to questions about selfcollection of vaginal swabs and self-testing with the OSOM kit are shown in Table 2. Most women reported that the instructions for getting a swab $(98.6 \% ; 145 / 147)$ and doing the OSOM test $(146 / 147 ; 99.3 \%)$ were clear and straightforward. For 127 (86.3\%) women, the swab collection and testing process was simple. The number of women who trust the OSOM test results was $135(91.8 \%)$, and 142 preferred selftesting to physician testing (96.5\%). 138 (93.8\%) participants 


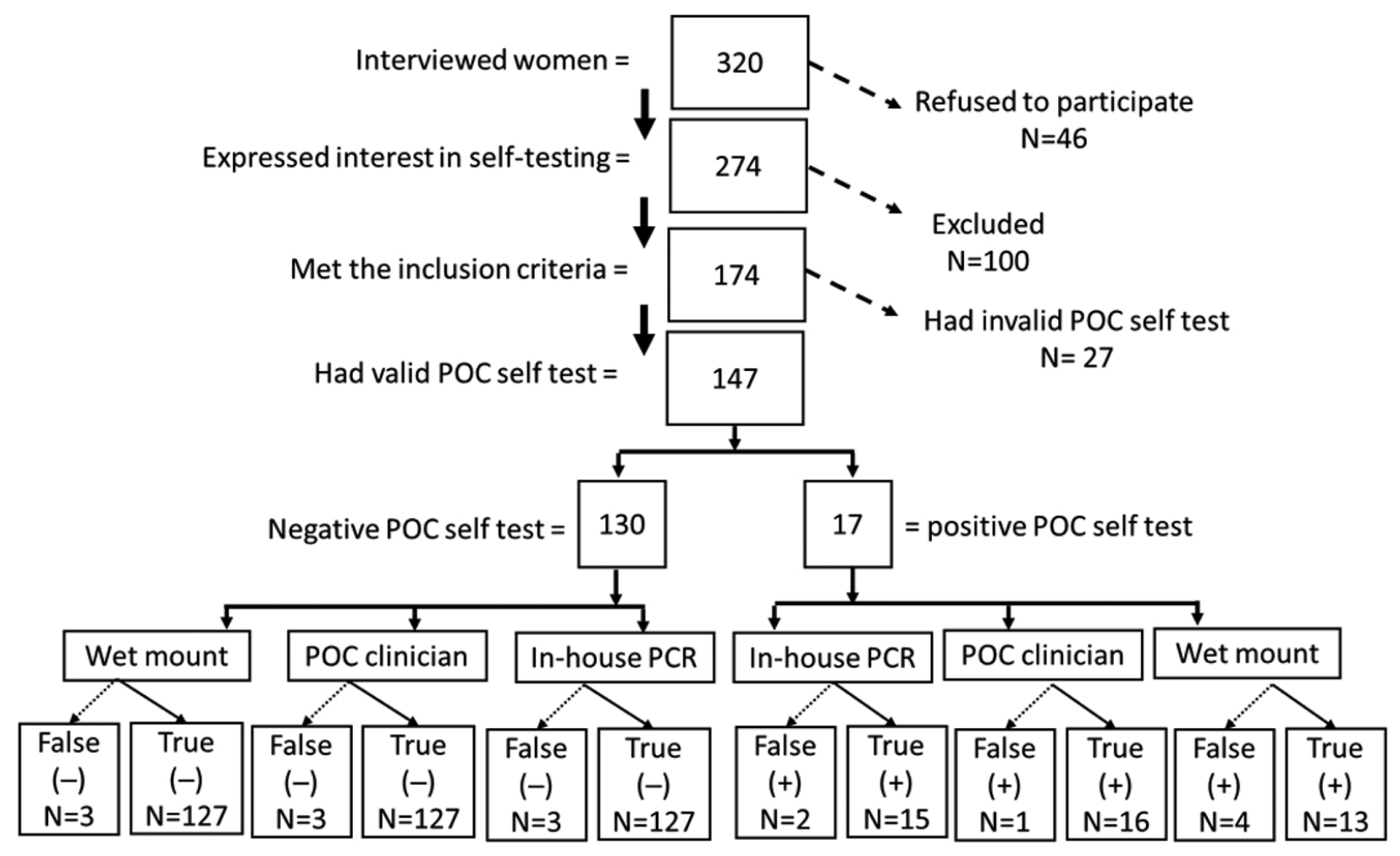

Figure 1. Women's enrollment and diagnostic testing results.

Table 1. The characteristics of the 147 women who took part in the study

\begin{tabular}{|c|c|c|c|c|}
\hline Variable & Category & Total (\%) & Trichomonas +(\%) & $p$ Value \\
\hline \multirow{2}{*}{ Age per years } & $18-25$ & $103(70.0)$ & 12 (11.6) & \multirow{2}{*}{0.73} \\
\hline & $26-35$ & $44(29.9)$ & $6(13.6)$ & \\
\hline \multirow[t]{2}{*}{ Nationality } & Saudi & $86(58.5)$ & $6(6.9)$ & \multirow{2}{*}{0.070} \\
\hline & Non-Saudi & $61(41.4)$ & $10(16.3)$ & \\
\hline \multirow[t]{2}{*}{ Current marital status } & Married & $108(73.4)$ & 14 (12.9) & \multirow{2}{*}{0.658} \\
\hline & Separated/Divorced/Widowed & $39(26.5)$ & $4(10.2)$ & \\
\hline \multirow[t]{4}{*}{ Education level $^{1}$} & Elementary & $10(6.8)$ & $8(80.0 \%)$ & \multirow{4}{*}{$<0.001^{* *}$} \\
\hline & Intermediate & 17 (11.5) & $3(17.6)$ & \\
\hline & Secondary & $23(15.6)$ & $5(21.7)$ & \\
\hline & Tertiary & 97 (65.9) & $2(2.0)$ & \\
\hline \multirow[t]{2}{*}{ Employment status } & Employed & $119(80.9)$ & $6(5.0)$ & \multirow{2}{*}{$<0.001^{* *}$} \\
\hline & Non-employed & $28(19.0)$ & $8(28.5)$ & \\
\hline \multirow[t]{2}{*}{ Residence } & Urban & 94 (63.9) & $11(11.7)$ & \multirow{2}{*}{0.789} \\
\hline & Rural & $53(36.0)$ & $7(13.2)$ & \\
\hline \multirow[t]{2}{*}{ Clinical symptoms ${ }^{2}$} & Absent & $28(19.0)$ & $6(21.4)$ & \multirow{2}{*}{0.099} \\
\hline & Present & $119(80.9)$ & $12(10.0)$ & \\
\hline
\end{tabular}

${ }^{1}$ There are four levels in Saudi Arabia's educational system: elementary (6 grades of primary school), intermediate ( 3 years following primary school), secondary (3 years of Qur'anic school, general school, or vocational school) and high level ( $\geq 3$ years of Bachelor's or graduate degree). ${ }^{2}$ Symptoms included abnormal vaginal discharge, genital itching, dysuria, lower abdominal pain and/or Malodour.

${ }^{* *}$ Highly significant difference observed among variable. 
Table 2. Participants' knowledge of STIs and attitudes toward OSOM self-testing

\begin{tabular}{|c|c|c|c|}
\hline Category & $\begin{array}{c}\text { Trichomonas }(+) \\
(\mathrm{n}=18) \\
\mathrm{n}(\%)\end{array}$ & $\begin{array}{c}\text { Trichomonas }(-) \\
(\mathrm{n}=129) \\
\mathrm{n}(\%)\end{array}$ & $\begin{array}{c}\text { Total } \\
(n=147)(\%)\end{array}$ \\
\hline \multicolumn{4}{|l|}{ Participants' knowledge and views towards STIs } \\
\hline Had never heard of the STIs before & $11(8.9)$ & $112(91.0)$ & $123(83.6)$ \\
\hline Not familiar with the STI prevention tools & $14(11.7)$ & $105(88.2)$ & 119 (80.9) \\
\hline Like to keep STI test results private & $16(13.6)$ & $101(86.3)$ & $117(79.5)$ \\
\hline Never heard of the Trichomonas rapid test & $9(6.1)$ & $137(93.8)$ & $146(99.3)$ \\
\hline Never done pregnancy test by her own & $13(14.9)$ & $74(85.0)$ & $87(59.1)$ \\
\hline \multicolumn{4}{|l|}{ Participants' perception towards OSOM self-testing } \\
\hline The instructions for getting a swab were clear & $5(3.4)$ & $140(96.5)$ & $145(98.6)$ \\
\hline The self-testing directions were straightforward & $8(5.4)$ & $138(94.5)$ & $146(99.3)$ \\
\hline It was easy to do self-collection and self-testing & $11(8.6)$ & $116(91.3)$ & $127(86.3)$ \\
\hline My test result appeared to be accurate & $13(9.6)$ & $122(90.3)$ & 135 (91.8) \\
\hline I prefer self- to clinic-collection vaginal swab specimen & $15(10.5)$ & $127(89.4)$ & $142(96.5)$ \\
\hline Others, in my opinion, should do the test on their own & $9(6.6)$ & 125 (91.9) & $136(92.5)$ \\
\hline If the test was offered over the counter, I would use it & $8(5.7)$ & $130(94.2)$ & $138(93.8)$ \\
\hline
\end{tabular}

STIs; sexually-transmitted infection, (+); Trichomonas vaginalis positive, (\%); Trichomonas vaginalis negative, (n); number.

Table 3. Performance characteristics of diagnostic tests for Trichomonas vaginalis in 147 women. In-house-PCR: 18 TP and 129 TN with overall prevalence of $12.2 \%$

\begin{tabular}{|c|c|c|c|c|c|c|c|c|c|}
\hline Test & TP & FP & TN & $\mathrm{FN}$ & $\begin{array}{l}\text { Sensitivity } \\
(95 \% \mathrm{CI})\end{array}$ & $\begin{array}{l}\text { Specificity } \\
(95 \% \mathrm{Cl})\end{array}$ & $\begin{array}{c}\text { Kappa } \\
\text { (\% agreement) }\end{array}$ & $\begin{array}{c}\text { PPV } \\
(95 \% \mathrm{Cl})\end{array}$ & $\begin{array}{c}\text { NPV } \\
(95 \% \mathrm{Cl})\end{array}$ \\
\hline Self OSOM & 15 & 2 & 127 & 3 & $\begin{array}{c}83.3 \% \\
(58.5-96.4)\end{array}$ & $\begin{array}{c}98.4 \% \\
(94.5-99.8)\end{array}$ & $0.83(96.5)$ & $\begin{array}{c}88.2 \% \\
(65.1-96.7)\end{array}$ & $\begin{array}{c}97.6 \% \\
(93.7-99.1)\end{array}$ \\
\hline Clinician OSOM & 16 & 0 & 129 & 2 & $\begin{array}{c}88.8 \% \\
(65.2-98.6)\end{array}$ & $\begin{array}{c}100.0 \% \\
(97.1-100.0)\end{array}$ & $0.93(98.6)$ & $100.0 \%$ (NA) & $\begin{array}{c}98.4 \% \\
(94.5-99.5)\end{array}$ \\
\hline Wet mount & 13 & 0 & 129 & 5 & $\begin{array}{c}72.2 \% \\
(46.5-90.3)\end{array}$ & $\begin{array}{c}100.0 \% \\
(97.1-100.0)\end{array}$ & $0.82(96.5)$ & $100.0 \%$ (NA) & $\begin{array}{c}96.2 \% \\
(92.4-98.1)\end{array}$ \\
\hline
\end{tabular}

POC: point-of-care, 95\% Cl: 95\% Confidence interval, TN: True negative, TP: True positive, FN: False negative, FP: False positive, NA: Not applicable, NPV: Negative predictive value, PPV: Positive predictive value.

Table 4. Agreement between the self-OSOM and Clinician-OSOM tests as well as the wet mount microscopy

\begin{tabular}{|c|c|c|c|c|c|c|c|c|c|}
\hline \multirow{2}{*}{$N=147$} & & \multicolumn{4}{|c|}{ Clinician-OSOM } & \multicolumn{4}{|c|}{ Wet mount } \\
\hline & & $P$ & $\mathrm{~N}$ & Total & $\begin{array}{c}\text { Kappa } \\
\text { (\% Agreement) }\end{array}$ & $P$ & $N$ & Total & $\begin{array}{c}\text { Kappa } \\
\text { (\% Agreement) }\end{array}$ \\
\hline \multirow[t]{3}{*}{ Self-OSOM } & $\mathrm{P}$ & 15 & 2 & 17 & \multirow{3}{*}{$\begin{array}{c}0.89 \\
(97.9 \%)\end{array}$} & 13 & 4 & 17 & \multirow{3}{*}{$\begin{array}{c}0.85 \\
(97.2 \%)\end{array}$} \\
\hline & $\mathrm{N}$ & 1 & 129 & 130 & & 0 & 130 & 130 & \\
\hline & Total & 16 & 131 & 147 & & 13 & 134 & 147 & \\
\hline
\end{tabular}

95\% Cl: 95\% Confidence interval, N: Negative, P: Positive.

stated they would use OSOM self-testing if it were available over the counter, and 136 (92.5\%) said they would recommend it to others.

\section{DISCUSSION}

The outcomes of our research reveal essential characteristics of Trichomonas infection in the study's population. The prevalence of infection was found to be relatively high $(\approx 12 \%)$ in the study population. An analysis of the number of $T$. vaginalis positive cases reported in Saudi Arabia over a five-year period revealed an average annual incidence of $10 \%$ per 100,000 Saudis (Madani, 2006). Outside of Saudi Arabia, prevalence rates varied by population, although the worldwide women's prevalence has been claimed to be $5-74 \%$ (Vos et al., 2012). Jones and colleagues detected infection with a $10 \%$ prevalence rate in South Africa and a $3 \%$ prevalence rate in Brazil based on PCR assay results (Jones et al., 2013). Crucitti et al. (2010) reported a prevalence of $\approx 33 \%$ for Trichomonas infection in self-collected vaginal swab specimens of 197 commercial sex workers using a PCR assay in a study done in Zambia. According to one study, the 
prevalence of Trichomonas infection in vaginal swabs of 734 Chinese female sex workers was determined to be $9.0 \%$ based on the results of microscopic inspection of wet mounts (Luo et al., 2016). According to the authors of this Chinese study, the prevalence in female sex workers could be underestimated due to the limited sensitivity of the wet mount techniques used. According to another study conducted in Mexico, Lopez-Monteon and colleagues have described a prevalence of $\approx 23 \%$ for Trichomonas infection in urine specimens of women at low risk for STIs using a PCR assay (López-Monteon et al., 2013). The prevalence of infection is influenced by factors such as access to health services, sexual behavior, hygiene practices, socioeconomic situations, and laboratory detection techniques (Miller et al., 2008).

Notably, Participant's age was not significantly associated with $T$. vaginalis infections although higher rates were seen in women aged 26-35 years, an age range associated with a lot of conception. This is in line with Nsagha et al. (2015) and not in line with Barbosa et al. (2020) findings. Also in our study, there was a significant correlation between women's education and work status and Trichomonas infection in the current study, with a higher risk of infection in women with a low educational level and who are unemployed, which is consistent with prior research (Abbai et al., 2013). Despite the OSOM self-testing has not yet been approved for use in asymptomatic women (Hobbs \& Seña, 2013), one-third of our participants who tested positive for trichomoniasis reported no vaginal symptoms. This finding highlights the need for Trichomonas screening guidelines considering the availability of reasonable treatments and serious health consequences of infection.

In our study, women were able to utilize the OSOM test on their own vaginal swab specimens and achieve higher sensitivity than wet mount microscopy. The wet mount was found to be less sensitive. Despite the literature's consensus on its limited sensitivity, direct observation of a pear-shaped trichomonad with its unique jerky or tumbling movement is considered $100 \%$ specific for Trichomonas infection (Vieira-Baptista et al., 2021). The main benefits of wet prep microscopy, the most often used diagnostic method for trichomoniasis, are its cost-effectiveness and speedy results, allowing for quick diagnosis and treatment. The low sensitivity adhered to the wet mounting may be attributed in most of cases to poor specimen storage or transportation conditions and/or to delayed examination (Stoner et al., 2013). For a positive wet-mount prep, the parasite must be motile, not only present in a specimen. Furthermore, microscopy has its own set of challenges in terms of training, experience, and equipment, and many clinicians have resorted to empirical diagnosis and treatment, resulting in poor infectious vaginitis management. Missed infections due to the low to moderate sensitivity of wet mount microscopy should not be overlooked, given the potential for Trichomonas to enhance the risk of HIV acquisition and/or transmission.

On patients-collected specimens, the OSOM test showed a sensitivity of $\approx 83 \%$. Huppert et al. (2010b) demonstrated sensitivity of $78 \%$ for the OSOM self-test in a study carried out elsewhere. The OSOM self- test has been evaluated in two developing nations, the sensitivity has been $\approx 83$ in one country and $\approx 68 \%$ in the other (Jones et al., 2013). In that study, Jones et al. have reported $\approx 77 \%$ pooled sensitivity for the OSOM self-test.

On the clinician-collected specimens, the OSOM test achieved sensitivity of $\approx 89 \%$ in our study. Earlier research has showed sensitivity ranged from $75 \%$ to $98 \%$ on clinicianobtained vaginal swabs, depending on the population group studied. The sensitivity of the OSOM test has been $75 \%$ in a
South African population (Garrett et al., 2019), 86\% in an Indian population (Madhivanan et al., 2013), 95\% in a Canadian population (Campbell et al., 2008), and $98 \%$ in a distinct Egyptian population (Zaki et al., 2011). Variations in OSOM test sensitivities were clearly due to changes in the prevalence of Trichomonas infection in the study population, as well as the gold standard used in each study. Regarding the test specificity, when used on self-collected swabs, the OSOM test exhibited a specificity of $98 \%$, and when used on clinician-collected swabs, it had a specificity of $100 \%$. The OSOM test demonstrated a fairly high specificity, which was comparable to that reported for it in several studies (Campbell et al., 2008; Madhivanan et al., 2013; Garrett et al., 2019).

The OSOM test was found to have a high level of agreement between clinician and patient-collected vaginal swabs in this study, going with a previous study (Khan et al., 2019). This high proportion of agreement indicates that the test findings are safe to some extent against swabbing and testing errors that could lead to an inaccurate diagnosis (Gaydos, 2018). Furthermore, the patient-obtained specimens ensure her comfort, convenience and help speeding up the clinical practice. According to the gold standard test, the OSOM self-test showed two false positives and one false negative result (s). It's possible that the false positive results are the consequence of sample contamination or an offtarget reaction (the OSOM test reacting with something present in the specimen other than $T$. vaginalis protein). Other studies have observed falsely positive OSOM test results, which supports our findings (Hegazy et al., 2012). Women in our study reported that the OSOM is a very simple to set up and operate. Unlike clinician-collected specimen, the OSOM self-testing was rated by most of our study participants as more comfortable, consistent with earlier studies (Huppert et al., 2010a). Reduced fear and shyness associated with selftesting contributed to women's preference for self-collection of samples over clinician-collection.

It is important to remember our research limitations. To begin, the OSOM self-testing was done solely in the clinic for the study's objectives, under the supervision of a female gynecologist, and in batches, which has been shown to be cost effective. Second, the population size might be regarded small and not a representative sample of the community for a study evaluating perception for a novel diagnostic approach. Third, even though self-testing findings were generally good, women who self-test in a clinic setting may have testing anxiety owing to the presence of the physician, even if she is female. Finally, the presence of the interviewer, as well as executing numerous tasks in the same location, may have an impact on survey respondents' responses. These constraints should be considered in future research. The OSOM self-testing approach in non-supervised settings (e.g. at home) and its cost-effectiveness in comparison to the existing amplification-based assay for Trichomonas should be the focus of future research in the study's population.

To summarize, the outcomes of our research reveal essential characteristics of $T$. vaginalis infection in the study's population. The prevalence of infection was found to be relatively high. Young age, low education, the existence of vaginitis symptoms, and unemployment were also identified as primary risk factors for infection in the study population. The OSOM self-test had a relatively good performance, outperforming the wet mount microscopy and comparable to the OSOM rapid test performed by the clinician. The majority of women accepted and preferred the strategy of selfcollection of their vaginal swabs over collection by the physician. Overall, our study's findings may have important 
consequences for the implementation of Trichomonas screening method based on OSOM self-testing in the study's population particularly during the Coronavirus pandemic.

\section{ACKNOWLEDGEMENTS}

The authors would like to acknowledge Taif University Researchers Supporting Project number (TURSP-2020/156), Taif University, Taif, Saudi Arabia for the financial support of the present research. The authors would also like to thank the patients who took part in the study. Lastly, the authors would also like to express their gratitude to the Director of KFMC and the head of the Microbiology laboratory for their ethical and reasonable collaboration.

\section{Conflict of interests}

The authors declare that they have no known competing financial interests or personal relationships that could have appeared to influence the work reported in this paper.

\section{REFERENCES}

Alderete, J.F., O'Brien, J.L., Arroyo, R., Engbring, J.A., Musatovova, O., Lopez, O., Lauriano, C. \& Nguyen, J. (1995). Cloning and molecular characterization of two genes encoding adhesion proteins involved in Trichomonas vaginalis cytoadherence. Molecular Microbiology 17: 69-83. https:// doi.org/10.1111/j.1365-2958.1995.mmi_17010069.x

Abbai, N.S., Wand, H. \& Ramjee, G. (2013). Sexually transmitted infections in women participating in a biomedical intervention trial in Durban: Prevalence, coinfections, and risk factors. Journal of Sexually Transmitted Diseases 2013: 358402. https://doi.org/10.1155/2013/358402

Barbosa, M.D.S., Andrade de Souza, I.B., Schnaufer, E.C.D.S., Silva, L.F.D., Maymone Gonçalves, C.C., Simionatto, S. \& Marchioro, S.B. (2020). Prevalence and factors associated with Trichomonas vaginalis infection in indigenous Brazilian women. PLOS ONE 15: e0240323. https://doi.org/10.1371/ journal.pone.0240323

Campbell, L., Woods, V., Lloyd, T., Elsayed, S. \& Church, D.L. (2008). Evaluation of the OSOM $^{\circledR}$ trichomonas rapid test versus wet prep exam for detection of $T$. vaginalis (TV) Vaginitis in women with a low prevalence of infection. Journal of Clinical Microbiology 46: 3467-3469. https://doi.org/ 10.1128/JCM.00671-08

Crucitti, T., Jespers, V., Mulenga, C., Khondowe, S., Vandepitte, J. \& Buvé, A. (2010). Trichomonas vaginalis is highly prevalent in adolescent girls, pregnant women, and commercial sex workers in Ndola, Zambia. Sexually Transmitted Diseases 37: 223-227. https://doi.org/10.1097/OLQ.0b013e 3181c21f93

Crucitti, T., Jespers, V., Mulenga, C., Khondowe, S., Vandepitte, J. \& Buvé, A. (2011). Non-sexual transmission of Trichomonas vaginalis in adolescent girls attending school in Ndola, Zambia. PLOS ONE 6: e16310. https://doi.org/10.1371/ journal.pone.0016310

Garber, G.E. (2005). The laboratory diagnosis of Trichomonas vaginalis. Canadian Journal of Infectious Diseases and Medical Microbiology 16: 35-38. https://doi.org/10.1155/2005/373920

Garrett, N., Mitchev, N., Osman, F., Naidoo, J., Dorward, J., Singh, R., Ngobese, H., Rompalo, A., Mlisana K. \& Mindel, A. (2019). Diagnostic accuracy of the Xpert CT/NG and OSOM Trichomonas Rapid assays for point-of-care STI testing among young women in South Africa: a cross-sectional study. BMJ Open 9: e026888. http://doi.org/10.1136/ bmjopen-2018-026888
Gaydos, C.A. (2018). Let's take a "selfie": Self-collected samples for STIS. Sexually Transmitted Diseases 45: 278-279. https://doi.org/10.1097/OLQ.0000000000000785

Hegazy, M.M., El-Tantawy, N.L., Soliman, M.M., El-Sadeek, E.S. \& El-Nagar, H.S. (2012). Performance of rapid immunochromatographic assay in the diagnosis of Trichomoniasis vaginalis. Diagnostic Microbiology and Infectious Disease 74: 49-53. https://doi.org/10.1016/j.diagmicrobio.2012.05.003

Herbst de Cortina, S., Bristow, C.C., Joseph Davey, D. \& Klausner, J.D. (2016). A systematic review of point of care testing for Chlamydia trachomatis, Neisseria gonorrhoeae, and Trichomonas vaginalis. Infectious Diseases in Obstetrics and Gynecology 2016: 4386127. https://doi.org/10.1155/2016/ 4386127

Hobbs, M.M. \& Seña, A.C. (2013). Modern diagnosis of Trichomonas vaginalis infection. Sexually Transmitted Infections 89: 434-438. http://doi.org/10.1136/sextrans-2013051057

Huppert, J., Hesse, E. \& Gaydos, C.A. (2010a). What's the point? How point-of-care STI tests can impact infected patients. Point of Care 9: 36-46. https://doi.org/10.1097/POC.0b013e $3181 \mathrm{~d} 2 \mathrm{~d} 8 \mathrm{cc}$

Huppert, J.S., Hesse, E., Kim, G., Kim, M., Agreda, P., Quinn, N. \& Gaydos, C. (2010b). Adolescent women can perform a point-of-care test for trichomoniasis as accurately as clinicians. Sexually Transmitted Infections 86: 514-519. https://doi.org/10.1136/sti.2009.042168

Jones, H.E., Lippman, S.A., Caiaffa-Filho, H.H., Young, T. \& van de Wijgert, J.H. (2013). Performance of a rapid self-test for detection of Trichomonas vaginalis in South Africa and Brazil. Journal of Clinical Microbiology 51: 1037-1039. https://doi.org/10.1128/JCM.01547-12

Khan, Z., Bhargava, A., Mittal, P., Bharti, R., Puri, P., Khunger, N. \& Bala, M. (2019). Evaluation of reliability of self-collected vaginal swabs over physician-collected samples for diagnosis of bacterial vaginosis, candidiasis and trichomoniasis, in a resource-limited setting: A crosssectional study in India. BMJ Open 9: e025013. https:// doi.org/10.1136/bmjopen-2018-025013

Lee, A.S. \& Cody, S.L. (2020). The Stigma of Sexually Transmitted Infections. The Nursing Clinics of North America 55: 295-305. https://doi.org/10.1016/j.cnur.2020.05.002

Lewis, D. (2014). Trichomoniasis. Medicine 42: 369-371. https:/ /doi.org/10.1016/j.mpmed.2014.04.004

López-Monteon, A., Gómez-Figueroa, F.S., Ramos-Poceros, G., Guzmán-Gómez, D. \& Ramos-Ligonio, A. (2013). Codetection of Trichomonas vaginalis and Candida albicans by PCR in urine samples in a low-risk population attended in a clinic first level in central Veracruz, Mexico. BioMed Research International 2013: 281892. https://doi.org/10.1155/ 2013/281892

Luo, L., Reilly, K.H., Xu, J.J., Wang, G.X., Ding, G.W., Wang, N. \& Wang, H.B. (2016). Prevalence and correlates of Trichomonas vaginalis infection among female sex workers in a city in Yunnan Province, China. International Journal of STD \& AIDS 27: 469-475. https://doi.org/10.1177/0956462415585449

Maciel, G.D.P., Tasca, T. \& De Carli, G.A. (2004). Clinical aspects, pathogenesis and diagnostic of Trichomonas vaginalis. Jornal Brasileiro de Patologia e Medicina Laboratorial 40: 152160. https://doi.org/10.1590/S1676-24442004000300005

Madani, T.A. (2006). Sexually transmitted infections in Saudi Arabia. BMC Infectious Diseases 6: 1-6. https://doi.org/ 10.1186/1471-2334-6-3 
Madhivanan, P., Li, T., Trammell, S., Desai, C., Srinivas, V., Arun, A., Klausner, J.D. \& Krupp, K. (2013). Performance of the OSOM Trichomonas Rapid Test for diagnosis of Trichomonas vaginalis infection among women in Mysore, India. Sexual Health 10: 320-324. https://doi.org/10.1071/SH13015

Manhart, L.E. (2017). M. genitalium on the loose: time to sound the alarm. Sexually transmitted diseases 44: 463-465. https:/ /doi.org/10.1097/OLQ.0000000000000665

Masha, S.C., Cools, P., Sanders, E.J., Vaneechoutte, M. \& Crucitti, T. (2019). Trichomonas vaginalis and HIV infection acquisition: A systematic review and meta-analysis. Sexually Transmitted Infections 95: 36-42. http://doi.org/ 10.1136/sextrans-2018-053713

Miller, M., Liao, Y., Gomez, A.M., Gaydos, C.A. \& D'Mellow, D. (2008). Factors associated with the prevalence and incidence of Trichomonas vaginalis infection among African American women in New York city who use drugs. The Journal of Infectious Diseases 197: 503-509. https://doi.org/ $10.1086 / 526497$

Nabweyambo, S., Kakaire, O., Sowinski, S., Okeng, A., Ojiambo, H., Kimeze, J., Najjingo, I. \& Bwanga, F. (2017). Very low sensitivity of wet mount microscopy compared to PCR against culture in the diagnosis of vaginal trichomoniasis in Uganda: A cross sectional study. BMC Research Notes 10: 1-6. https://doi.org/10.1186/s13104-017-2581-1

Napoleon, S.C., Maynard, M.A., Almonte, A., Cormier, K., Bertrand, T., Ard, K.L. \& Chan, P.A. (2020). Considerations for STI clinics during the COVID-19 pandemic. Sexually Transmitted Diseases 47: 431-433. https://doi.org/10.1097/ OLQ.0000000000001192

Nsagha, D.S., Zofou, D., Assob, J.N., Njunda, A.L., Nchang, C.D., MvoNgum, N., Patrick, W.E. \& Marcelin, N.N. (2015). The epidemiology of Trichomonas vaginalis, Gardnerella vaginalis and Candida albicans co-infections in women attending the Yaounde University teaching hospital. American Journal of Epidemiology and Infectious Disease 3: 28-31. https:// doi.org/10.12691/ajeid-3-2-2
Stoner, K.A., Rabe, L.K., Meyn, L.A. \& Hillier, S.L. (2013). Survival of Trichomonas vaginalis in wet preparation and on wet mount. Sexually Transmitted Infections 89: 485-488. http:// doi.org/10.1136/sextrans-2012-051001

Vieira-Baptista, P., Grinceviciene, Š., Oliveira, C., FonsecaMoutinho, J., Cherey, F. \& Stockdale, C.K. (2021). The international society for the study of vulvovaginal disease vaginal wet mount microscopy guidelines: How to perform, applications, and interpretation. Journal of Lower Genital Tract Disease 25: 172-180. https://doi.org/10.1097/lgt. 0000000000000595

Vos, T., Flaxman, A.D., Naghavi, M., Lozano, R., Michaud, C., Ezzati, M., Shibuya, K., Salomon, J.A., Abdalla, S., Aboyans, V. et al. (2012). Years lived with disability (YLDs) for 1160 sequelae of 289 diseases and injuries 1990-2010: a systematic analysis for the Global Burden of Disease Study 2010. The lancet 380: 2163-2196. https://doi.org/ 10.1016/S0140-6736(12)61729-2

World Health Organization. (2020). WHO recommendations on self-care interventions: Self-collection of samples for sexually transmitted infections (STIs) (No. WHO/SRH/ 20.10). World Health Organization 2020.

Zaki, M.A., Moussa, H.M. \& Hassanin, O.M. (2011). Evaluation of the OSOM Trichomonas rapid test for detection of trichomoniasis vaginalis. Parasitologists United Journal 4: 177-184. 